\title{
Revealing novel quantum phases in quantum antiferromagnets on random lattices
}

\author{
R. Yu ${ }^{1,2}$, S. Haas $^{3}$, T. Roscilde ${ }^{4}$ \\ 1 Department of Physics and Astronomy, University of Tennessee, Knoxville, TN 37996, USA \\ 2 Material Science and Technology Division, Oak Ridge National Laboratory, Oak Ridge, TN 32831, USA \\ 3 Department of Physics and Astronomy, University of Southern California, Los Angeles, CA 90089-0484, USA \\ ${ }^{4}$ Laboratoire de Physique, École Normale Supérieure de Lyon, 46 Allée d'Italie, 69003 Lyon, France
}

Received May 5, 2009

\begin{abstract}
Quantum magnets represent an ideal playground for the controlled realization of novel quantum phases and of quantum phase transitions. The Hamiltonian of the system can be indeed manipulated by applying a magnetic field or pressure on the sample. When doping the system with non-magnetic impurities, novel inhomogeneous phases emerge from the interplay between geometric randomness and quantum fluctuations. In this paper we review our recent work on quantum phase transitions and novel quantum phases realized in disordered quantum magnets. The system inhomogeneity is found to strongly affect phase transitions by changing their universality class, giving the transition a novel, quantum percolative nature. Such transitions connect conventionally ordered phases to unconventional, quantum disordered ones - quantum Griffiths phases, magnetic Bose glass phases - exhibiting gapless spectra associated with low-energy localized excitations.
\end{abstract}

Key words: Heisenberg antiferromagnets, quantum disorder, geometric randomness, percolation, Bose glass

PACS: $75.10 . J \mathrm{~m}, 75.10 . \mathrm{Nr}, 75.40 . \mathrm{Cx}, 64.60 . \mathrm{Ak}$

\section{Introduction}

Quantum phase transitions (QPTs) and related collective quantum phases represent one of the most exciting research topics in condensed matter physics [1]. Contrary to thermal phase transitions, QPTs occur at zero temperature upon tuning a parameter of the system Hamiltonian. The emergence of quantum collective phenomena opens the path towards quantum phases that do not admit any classical counterpart. Quantum magnets provide a large showcase of materials in which QPTs have been experimentally demonstrated. Quantum fluctuations, driving the system through a QPT, can be continuously tuned by e. g. applying a magnetic field or by exerting pressure on the sample to control the magnetic couplings among the spins. A well-known example is represented by magnetic Bose-Einstein condensation (BEC) in spin-gap materials [2], such as systems of weakly coupled $S=1 / 2$ dimers. These magnetic insulators show a paradigmatic quantum-disordered ground state, namely a total singlet state with a gap to all triplet excitations. Application of a magnetic field can close the triplet gap, inducing the appearance of bosonic spin triplets in the ground state of the system, forming a Bose condensate of magnetic quasiparticles. This condensed state corresponds to a magnetically ordered state with spontaneous appearance of a staggered magnetization transverse to the field.

A completely different route towards QPT transitions in quantum magnets is represented by the effect of non-magnetic doping. Starting from a magnetically ordered state, a simple route towards disordering the system is by diluting the lattice via site or bond removal. A genuine quantum phase transition is realized if quantum fluctuations lead to the loss of magnetic order at $T=0$ before the lattice reaches the percolation threshold. Experimental studies on model magnets, such as non-magnetically doped $\mathrm{La}_{2} \mathrm{CuO}_{4}$ [3], supported by extensive numerical studies [4] show that fundamental quantum models like the two-dimensional quantum Heisenberg antiferromagnet 
do not develop strong enough quantum fluctuations for a quantum phase transition to occur. Yet, increasing quantum fluctuations $a d$ hoc, e. g. by explicit lattice dimerization [5-7] or by anisotropic bond dilution [8], can lead indeed to disorder-induced quantum phase transitions (see figure 1). And what better strategy could one envision to tune quantum fluctuations than to consider a system which already exhibits a quantum phase transition in the clean limit?

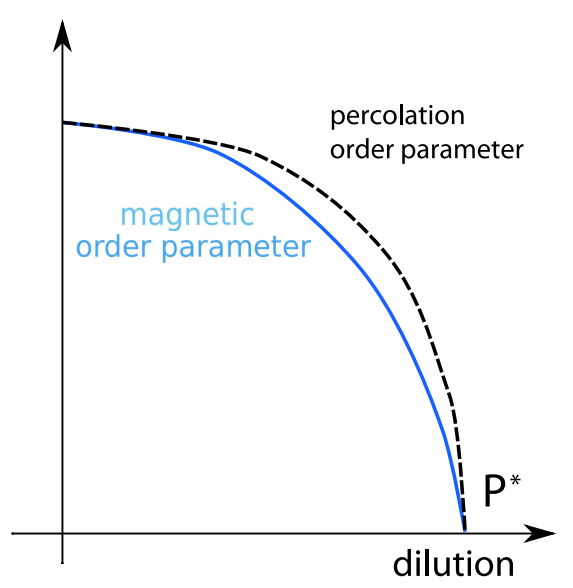

(a)

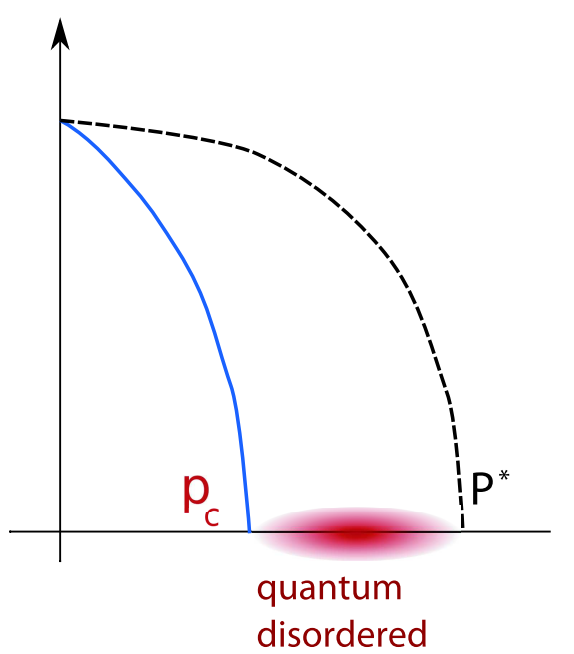

(b)

Figure 1. (a) Conventional percolation transition in a magnet: upon diluting the system the magnetic order parameter is found to vanish at the percolation threshold $p^{*}$. (b) Disorderinduced quantum phase transition: at a critical dilution $p_{\mathrm{c}}<p^{*}$ the magnetic order disappears, leaving space to a novel quantum disordered phase.

This idea brings naturally to the investigation of the effect of disorder on quantum critical phenomena in magnetic systems, and in particular on magnetic BEC as a paradigmatic magnetic quantum phase transition [9-12]. Introducing disorder in the magnetically ordered phase immediately close to the condensation QPT corresponds to exposing the dilute gas of spin triplets induced by the field to a random potential: as in any system of weakly interacting quantum particles, this leads to Anderson localization for moderate (or even infinitesimal) disorder strengths [13]. This property translates into the possibility of disrupting the field-induced magnetic order by diluting the lattice well below the percolation threshold, accomplishing in this way a genuine disorder-induced quantum phase transition.

A side effect of doping weakly coupled dimer systems is the appearance of unpaired $S=1 / 2$ local moments, which are coupled through virtual excitations of the intact dimers $[11,14,15]$ (see figure 2a). The resulting random network of interacting local moments can support long-range antiferromagnetic order in zero field, giving rise to a counter-intuitive phenomenon of order by disorder (OBD). This ordered state is found to be disrupted by the application of a moderate magnetic field $[11,12]$. The resulting disordered phase shows short-range antiferromagnetic correlations surviving on localized regions, which can be mapped onto Anderson localized quasiparticles.

Hence we see in general that disorder-induced quantum phase transitions drive the system to novel quantum disordered phases with rather exotic properties. A common denominator of these phases is a gapless spectrum associated with the appearance of low-energy localized excitations, associated with exponentially rare regions in the disordered lattice. If the energy cost of the localized excitation decreases algebraically with the inverse size of the region that hosts it, all conventional response functions remain non-singular in this phase down to $T=0$. On the other hand, if the same energy cost decreases exponentially when the size of the region increases, this leads to quantum Griffiths singularities [16] with a non-universal, disorder-dependent divergent behavior in measurable quantities such as the uniform susceptibility.

In this paper we review our recent progress in studying disorder-induced QPTs as well as 


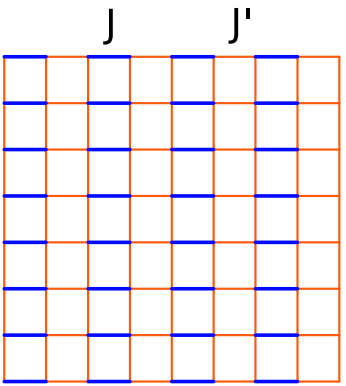

(a)

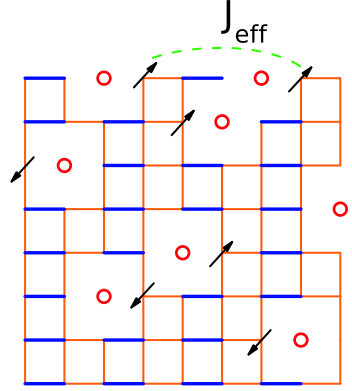

(b)

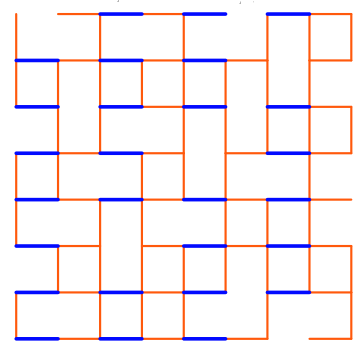

(c)

Figure 2. (a) A square lattice decomposed into dimer and inter-dimer bonds. The dimer bonds are shown in dark color with coupling $J$, and the inter-dimer bonds are shown in light color with coupling $J^{\prime}$. Both $J$ and $J^{\prime}$ are antiferromagnetic. (b) Site dilution of the dimer singlet ground state releases random magnetic local moments which are coupled through long-range coupling $J_{\text {eff }}$. The circles refer to the non-magnetic impurities, and arrows correspond to local moments near the impurity sites. (c) Inhomogeneous bond dilution of the same lattice: the dimer bonds $(J)$ are populated with probability $P$, while the inter-dimer bonds $\left(J^{\prime}\right)$ are populated with probability $P^{\prime}$.

the effect of disorder on field-induced QPTs in two-dimensional quantum magnets. This topic is particularly challenging from a technical point of view. Indeed conventional perturbation methods are generally doomed to failure at quantum critical points; renormalization group schemes, while generally successful for the study of critical points in clean systems, prove to be particularly hard for critical points in presence of disorder [17]. A third way is provided by numerically exact methods, which are particularly well developed for unfrustrated quantum spin systems. In particular we make use of the stochastic series expansion quantum Monte Carlo method [18] which proves to be a very powerful quantum Monte Carlo technique to investigate the low-temperature properties of quantum spin systems on a large scale. The non-local nature of the update algorithm makes it possible to circumvent critical slowing down and to investigate quantum critical phenomena in a very accurate way. While disorder imposes the extra cost of averaging over disorder statistics, this task can be accomplished on modern supercomputers.

The structure of the paper is as follows. In section 2 we show how the interplay between anisotropic geometric randomness and quantum fluctuations gives rise to a new class of percolative quantum phase transitions in an inhomogeneous bond-diluted antiferromagnet in two dimensions (2D). This transition opens a novel quantum disordered phase which exhibits quantum Griffiths singularities. In section 3, we analyze the very rich phase diagram of site-diluted weakly coupled dimer systems in a magnetic field, showing the occurrence of an extended magnetic Bose glass phase.

\section{Percolative quantum phase transition in strongly fluctuating quantum antiferromagnets}

Our starting point is the quantum Heisenberg antiferromagnetic model defined on a regular bipartite lattice, as illustrated in figure 2a, with Hamiltonian

$$
\mathcal{H}=\sum_{\langle i j\rangle} J_{i j} \boldsymbol{S}_{i} \cdot \boldsymbol{S}_{j}+\sum_{\langle l m\rangle} J_{l m}^{\prime} \boldsymbol{S}_{l} \cdot \boldsymbol{S}_{m}-g \mu_{b} H \sum_{i} S_{i}^{z},
$$

where $S$ are $S=1 / 2$ spin operators. In the most general case, two sets of bonds with different local strengths $\left(J_{i j}\right.$ and $\left.J_{l m}^{\prime}\right)$ have been singled out. In the clean case one has $J_{i j}=J$ for all $\langle i j\rangle$ and $J_{l m}^{\prime}=J^{\prime}$ for all $\langle l m\rangle$. In the following we will focus our attention on 1) the case of a planar array of dimers in a square lattice (as specifically illustrated by figure 2a) and 2) the case of a bilayer system, in which the bonds of strength $J^{\prime}$ form two square lattices which are connected by 
the bonds of strength $J$. In the case 1) of a simple square lattice, the choice $J=J^{\prime}$ reproduces the well-known limit of the two-dimensional quantum Heisenberg antiferromagnet (2DQHAF). In this section we will consider the case of zero applied magnetic field $H$, while the effects of a finite field will be accounted for in the next section.

In the clean limit, the 2DQHAF is well known to support long-range Néel order[19]. As mentioned in the introduction, a central question of quantum magnetism is the response of magnetically ordered systems to the dilution of their magnetic lattice, either in the form of bond or site dilution. From a geometric point of view, bond or site dilution reduce the connectivity of the lattice, ultimately leading to a percolative phase transition [20] beyond which the system is broken up into finite clusters. In a classical spin system, this percolation transition is coupled to a magnetic transition with the same critical exponents, since spontaneous magnetic order cannot survive beyond the percolation threshold. In a quantum spin system, on the other hand, a progressive reduction of the lattice connectivity enhances quantum fluctuations in a continuous fashion, raising the possibility of quantum destruction of magnetic order before the percolation threshold is reached. Recently, the evolution of the magnetic state of the 2DQHAF under site or bond dilution has been studied extensively both in experiment and theory. Experimentally, the effect of site dilution has been probed in $\mathrm{La}_{2} \mathrm{Cu}_{1-p}(\mathrm{Zn}, \mathrm{Mg})_{p} \mathrm{O}_{4}$, in which magnetic $\mathrm{Cu}^{2+}$ ions are replaced randomly by non-magnetic $\mathrm{Zn}^{2+}$ or $\mathrm{Mg}^{2+}$ ions [3]. The fundamental result of this study is that magnetic order at low temperature disappears at a critical dilution $p=p_{\mathrm{c}}$ which coincides with the percolation threshold $p^{*}=0.40725[20]$. This has been further confirmed by extensive numerical simulations both in the case of site and bond dilution [4], showing that the percolating cluster supports long-range order up to the percolation threshold; this means that magnetism can only be discarded geometrically by fragmenting the percolating cluster beyond $p^{*}$.

An alternative scenario to the above classical percolation picture is offered by quantum percolation, in which the geometric transition and the magnetic one are decoupled by quantum fluctuations. This scenario invokes the fact that spins involved in locally strongly fluctuating quantum states, such as dimer singlets and resonating valence bond states, are weakly correlated with the remainder of the system. In a random network of spins, the local strongly fluctuating states create weak links with small spin-spin correlations. If these weak links are part of the backbone of the percolating cluster, they can prevent the percolating cluster from developing long-range order. Therefore, if lattice dilution favors the local formation of such states, it is possible to drive the system towards a quantum disordered state before the percolation threshold is reached, decoupling percolation from magnetic ordering.

This quantum percolation scenario has been recently demonstrated for the $S=1 / 2$ Heisenberg model on the bilayer lattice under dimer dilution [5-7]. In this model quantum fluctuations can be arbitrarily tuned by increasing the strength of the inter-layer coupling $J$ with respect to the intra-layer one $J^{\prime}$ : indeed, even in the clean model, for $J>J_{\mathrm{c}}=2.5 J^{\prime}$ the spins on each inter-layer dimer form a singlet and long-range order is lost [21]. Randomly diluting the bilayer by taking away a percentage $p$ of whole dimers at once leads to the percolation of the bilayer lattice for $p=p^{*}$, analogously to what happens on a simple square lattice. For $J \lesssim J^{*}=0.16 J^{\prime}$ the bilayer shows long-range order up to the percolation threshold [6]. The tuning knob of quantum fluctuations offered by the inter-layer coupling allows to destroy long-range order on the percolating cluster for $J \gtrsim J^{*}$ : beyond this value, geometric percolation of the lattice is no longer a sufficient condition for long-range magnetic ordering, due to the extremely strong quantum fluctuations on dimers with lower local connectivity. The quantum disordered phase appearing on a percolated lattice of dimers still lacks a complete characterization, but it is legitimate to suspect that, for $J<J_{\text {c }}$, rare but arbitrarily large regions which are devoid of dimer vacancies can support locally magnonlike excitations similar to those of the clean system in its magnetically ordered phase. Hence the spectrum of the system is expected to be gapless, but not leading to a singular contribution to the response functions (as shown by the regular behavior of the uniform susceptibility in [6]). A very similar picture of quantum percolation has been found by two of us in the anisotropic $S=1$ Heisenberg antiferromagnet with site dilution [22]: here the quantum disordered phase has been identified with a Mott glass, namely a phase with a gapless spectrum and a vanishing 
compressibility.

In [8] we have shown that a similar quantum percolation scenario can be achieved in the standard 2DQHAF under inhomogeneous bond dilution. In our model all bonds are of equal strength $J=J^{\prime}$, and quantum fluctuations are instead enhanced in a purely geometrical fashion by the lattice randomness. As illustrated in figure 2a, a square lattice can be geometrically decomposed into dimers and ladders (made of inter-dimer bonds) in such a way that there are no two adjacent dimers or ladders. The inhomogeneous bond dilution is realized by assigning different occupation probabilities to intra-dimer and inter-dimer bonds. Explicitly

$$
\begin{aligned}
& \text { dimer bonds } J_{i j}= \begin{cases}J & \text { with probability } P, \\
0 & \text { with probability } 1-P,\end{cases} \\
& \text { ladder bonds } J_{l m}^{\prime}= \begin{cases}J & \text { with probability } P^{\prime}, \\
0 & \text { with probability } 1-P^{\prime} .\end{cases}
\end{aligned}
$$

As it is well-known, $S=1 / 2$ spin ladders have a quantum disordered singlet ground state [23], and the same applies to weakly connected dimer lattices, such as the comb lattice. This special property of the $S=1 / 2$ Heisenberg model on low-dimensional lattices is the key of our dilution scheme. In fact, inhomogeneous bond dilution $P \neq P^{\prime}$ (see figure 2c) favors the appearance of ladder segments $\left(P<P^{\prime}\right)$ or of weakly connected dimers $\left(P>P^{\prime}\right)$, both of which have the tendency to support locally a strongly fluctuating quantum state with a significant singlet component, and effectively decoupled from the rest of the percolating cluster. When such geometrical structures appear on the backbone of the percolating cluster, they can effectively break it into sub-clusters from the point of view of magnetic correlations, leading to a quantum-disordered ground state.

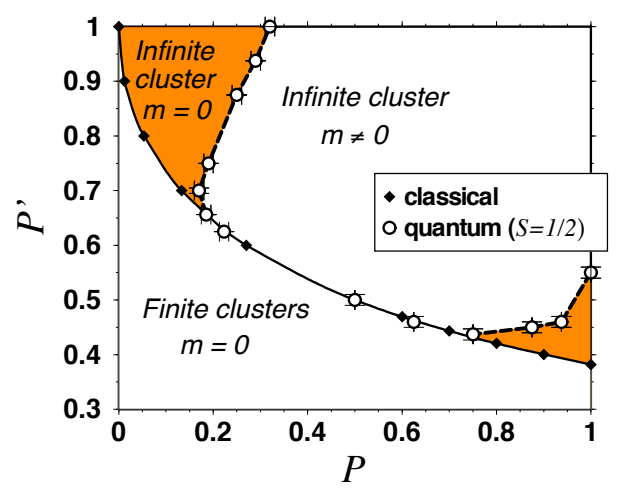

Figure 3. Phase diagram of the inhomogeneously bond-diluted $S=1 / 2$ antiferromagnet on the square lattice. The colored area indicates the quantum-disordered region in which the system has developed an infinite percolating cluster, but the magnetization $m$ of the system vanishes because of quantum fluctuations.

The phase diagram of this inhomogeneous bond dilution model, as resulting from an extensive classical and quantum Monte Carlo study [8], is presented in figure 3. When the inhomogeneity is weak $\left(P \approx P^{\prime}\right)$, the magnetic transition is found to coincide with the percolation one, both in terms of the location of the transition and in terms of its critical exponents. This is in agreement with the findings in the homogeneous limit $P=P^{\prime}$ already investigated in [4]. However, for strong enough inhomogeneity the magnetic transition deviates from the geometric one, turning into a quantum phase transition beyond two multi-critical points. The critical exponents, extracted from a finitesize scaling analysis of the correlation length, confirm this crossover from classical to quantum percolation: $\nu=4 / 3$ and $z=1.9 \approx D=91 / 48$ (the fractal dimensions of the percolation cluster at threshold [20]) are found for weak to intermediate inhomogeneity, while $\nu=1$ and $z=1$ are found for sufficiently strong inhomogeneity [8].

As seen in figure 3, the bifurcation of the magnetic transition line with respect to the percolation transition line at strong enough inhomogeneity opens up two quantum disordered phases, 
characterized by the existence of an infinite percolating clusters but zero staggered magnetization $m$. Real-space spin-spin correlations in this phase are short-ranged [24]. But the perfectly correlated disorder in imaginary time allows for long-range correlations in this extra dimension, giving rise to the so-called quantum Griffiths singularities [16]. As already mentioned in the introduction, the dilution of the lattice introduces local $S=1 / 2$ moments. If one dilutes a lattice developing a spin-gapped singlet ground state with a finite correlation length $\xi_{0}$ (as it will be the case in the following section), or if dilution induces the local formation of such singlet states (as it is the case in this section), the resulting local moments develop mutual effective interactions via the exchange of virtual gapped excitations of the intermediate regions. The effective couplings, $J_{\text {eff }}$, decay exponentially with the distance, as a result of the finite "mass" of the exchanged excitation. For two sites $i$ and $j$ with positions $\boldsymbol{r}_{i}, \boldsymbol{r}_{j}$, we have that $[11,14,15]$

$$
J_{\mathrm{eff}}(i, j) \sim J(-1)^{\boldsymbol{r}_{i}-\boldsymbol{r}_{j}} \exp \left(-\left|\boldsymbol{r}_{i}-\boldsymbol{r}_{j}\right| / \xi_{0}\right) .
$$

It is important to notice that the staggering prefactor eliminates any frustration effect. At variance with site dilution (compare figure $2 \mathrm{~b}$ ), for bond dilution these local $S=1 / 2$ moments always occur in pairs. The effective interaction between two closeby moments is the strongest $(\sim J)$, so that the lowest-energy excitations are not associated with exciting a pair of neighboring moments, but rather exciting two pairs of such moments lying far apart from each other by rotating one pair with respect to the other (see figures $4 \mathrm{a}, 4 \mathrm{~b}$ ). According to equation (4), this excitation has an

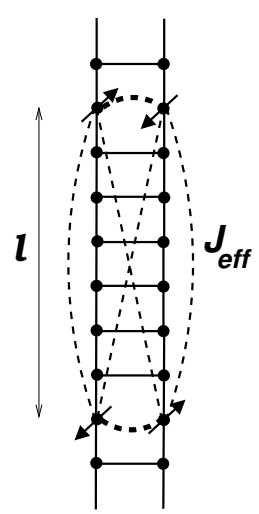

(a)

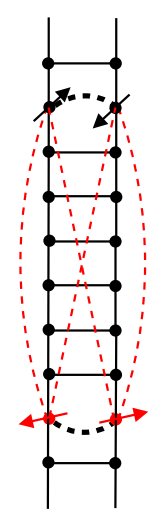

(b)

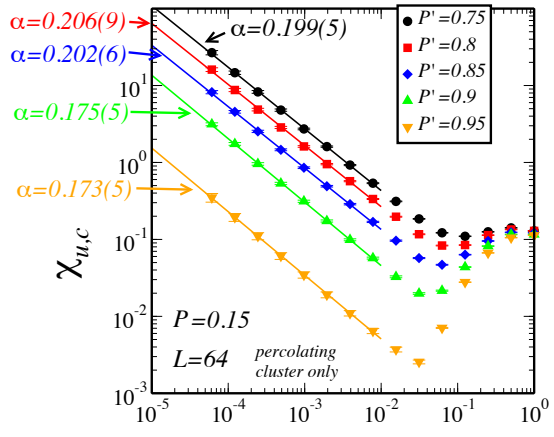

(c) $T / J$

Figure 4. (a)-(b) Sketch of a low-energy excitation in the bond-diluted ladder system. (c) Low-temperature uniform susceptibility of the percolating cluster only, $\chi_{u, c}$, in the quantumdisordered (ladder-like) regime. The solid lines are power-law fits of the form $\chi_{u, c} \sim T^{-1+\alpha}$, and the resulting fit coefficients $\alpha$ are indicated. The percolating cluster is picked as the largest cluster in a $64 \times 64$ lattice. The non-universal $\alpha$ values indicate the existence of Griffiths singularities in this quantum-disordered phase.

energy scaling exponentially to zero with the inter-pair distance $l$, but the probability of existence of two pairs separated by a large distance is conditioned by the presence of a clean region in between them, whose probability in a diluted system is also exponentially suppressed with its size. In the case depicted in figures $4 \mathrm{a}$, $4 \mathrm{~b}$, this probability is $\left(P^{\prime}\right)^{n l}=\exp \left(-n\left|\ln P^{\prime}\right| l\right.$ ) (where $n$ is the characteristic number of bonds present in a region of linear size $l$ ). The presence of an exponentially rare local excitation with exponentially small energy leads to a cancellation of the two exponentials in the calculation of the contribution of such excitations to fundamental response functions, such as the uniform susceptibility. The result is a paradigmatic quantum Griffiths effect [24]. Indeed the uniform susceptibility of the percolating cluster in the quantum disordered phase displays a non-universal power-law divergence when $T \rightarrow 0$, as presented in figure 4c. It is important to stress that here we only consider percolating clusters with an even number of particles, which means that the ground state on a finite-size system is expected to be a total spin singlet. Hence the presence of a non-universal divergence of the uniform susceptibility is not due to the presence of paramagnetic 
dangling spins, but it is a genuine result of the fact that the spectrum over the singlet ground state is gapless.

From an experimental point of view, bond dilution can be considered as a limit of bond disorder introduced by doping the non-magnetic atoms/ions lying on the super-exchange paths responsible for the occurrence of antiferromagnetic couplings. Hence inhomogeneous bond disorder can be introduced in antiferromagnets in which super-exchange paths are mediated by different atomic species along different spatial directions. This is the case of many spin-ladder compounds, such as $\left(\mathrm{C}_{5} \mathrm{H}_{12} \mathrm{~N}_{2}\right)_{2} \mathrm{CuBr}_{4}$, in which intra-ladder and inter-ladder couplings between $\mathrm{Cu}^{2+}$ ions are mediated by chemically different non-magnetic ions. From a more fundamental point of view, our results, along with those on dimer-diluted lattices [5-7] and on diluted anisotropic $S=1$ models $[22]$ show that disorder-induced magnetic transitions can be pushed arbitrarily far from percolation thresholds.

\section{Field-induced quantum disordered states in site-diluted lattices}

In the previous section, we have seen that lattice dilution can locally enhance quantum fluctuations in a quantum magnet, possibly discarding long-range order. In this section the critical role of quantum fluctuations will be tuned arbitrarily by driving the system close through a quantum phase transition in presence of disorder.

As already mentioned in the introduction, the quantum phase transition in question is represented by magnetic Bose-Einstein condensation in a system of weakly coupled $S=1 / 2$ dimers in a magnetic field. As in the previous section, we consider two different geometries leading to essentially the same physics: a bilayer geometry with interlayer couplings $J$ and intra-layer couplings $J^{\prime}$; a planar dimer array (compare figure 2a), with dimer couplings $J$ and inter-dimer couplings $J^{\prime}$. In the absence of lattice dilution, for both geometries, a ratio of couplings $J / J^{\prime}$ overcoming a critical value $\left(J / J^{\prime}\right)_{\mathrm{c}}(\approx 2.5$ for the bilayer system, $\approx 1.91$ for the planar dimer system [25]) stabilizes a quantum disordered ground state with dimer singlets on the strongest magnetic bonds. In both systems, the application of a magnetic field $h=g \mu_{b} H / J$ (in reduced units) leads to condensation of triplet excitations at a lower critical field $h_{\mathrm{c} 1}^{(0)}$ and to the appearance of staggered magnetic order transverse to it [2]. Increasing the field up to an upper critical field $h_{\mathrm{c} 2}^{(0)}$ leads to full polarization of the spins and to the destruction of spontaneous order.

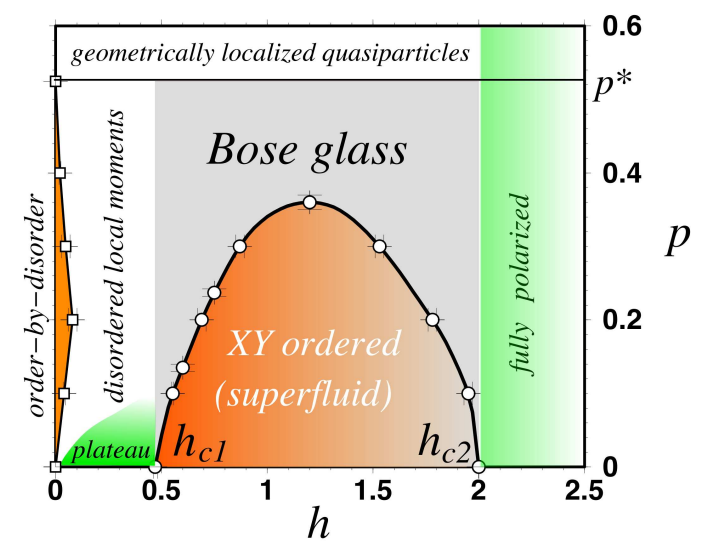

Figure 5. Ground-state phase diagram of the site-diluted bilayer Heisenberg antiferromagnet with $J / J^{\prime}=4$ in the field-dilution plane. Ordered phases are indicated in orange, gapped disordered phases are indicated in green, and gapless disordered phases in white and grey.

Doping the system induces a very rich phase diagram at $T=0$, as illustrated in figure 5 . If the precise location of the phase boundaries depends on the model parameter $J / J^{\prime}$ as well as on the lattice geometry, the qualitative features of this phase diagram are actually independent of the 
value of $J / J^{\prime}$, as long as $J / J^{\prime}>\left(J / J^{\prime}\right)_{\mathrm{c}}$, and of the specific geometry of the dimer array (either bilayer or planar array or other unfrustrated lattices). For the specific case shown in figure 5 (bilayer system with $J / J^{\prime}=4$ ), the clean limit $p=0$ features Bose-Einstein condensation transitions at $h_{\mathrm{c} 1}^{(0)} \approx 0.5$ and $h_{\mathrm{c} 2}^{(0)}=2$. Introducing site dilution of the bilayer lattice leads to an upward shift of the lower critical field $h_{\mathrm{c} 1}$ with respect to its clean value $h_{\mathrm{c} 1}^{(0)}$, and a downward shift of the upper critical field. This means that diluting the system at fixed field starting from its ordered phase $h_{\mathrm{c} 1}^{(0)}<h<h_{\mathrm{c} 2}^{(0)}$ leads to a disorder-induced transition into a novel quantum disordered phase. This all happens well away from the geometrical percolation threshold $p^{*}$, which means that the disorder-induced transition is a genuine quantum phase transition.

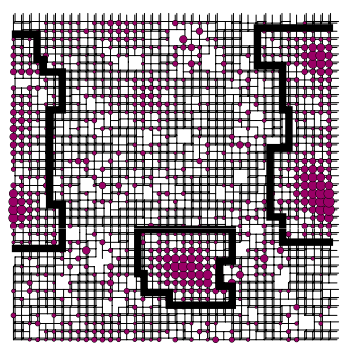

(a)

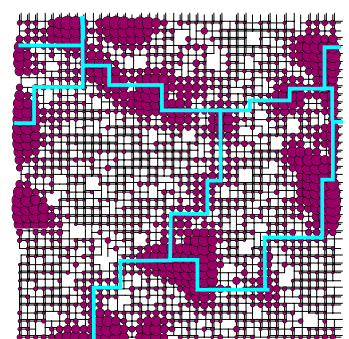

(b) $\bullet \mathrm{m}=0.05$

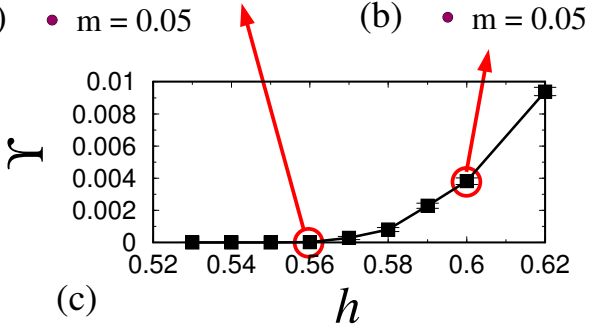

Figure 6. (a)-(b): Real-space images of the dimer magnetization $m_{i}=\left\langle S_{i, 1}^{z}+S_{i, 2}^{z}\right\rangle$ on intact dimers in a $40 \times 40 \times 2$ bilayer with $J / J^{\prime}=4$, dilution $p=0.1$ and at inverse temperature $\beta J=256$, for $h=0.56$ (a) and $h=0.6$ (b). The radius of the dots is proportional to the dimer magnetization. The magnetization of unpaired spins is omitted for clarity. The most visible localized states are highlighted in (a), while the backbone of the percolating magnetized network is highlighted in (b). (c) Superfluid density (spin stiffness) as a function of the field for the specific sample considered.

The novel phase that opens up at the disorder-induced transition can be fully characterized in terms of bosonic quasiparticles. In the case of $h \gtrsim h_{\mathrm{c} 1}^{(0)}$ these quasi-particles (QPs) are represented by the dilute triplet gas induced by the field, condensing in the magnetically ordered phase. The case $h \lesssim h_{\mathrm{c} 2}^{(0)}$ is the (approximately) particle-hole symmetric one, in which singlet quasi-holes (QHs) form a dilute gas that Bose condenses. The introduction of site dilution in the system creates an effective random potential for QPs (analogous considerations apply to QHs). Indeed site dilution leads to the disappearance of whole dimers hosting a QP, or to the appearance of dangling spins (local moments) which are essentially all polarized in the field range $h_{\mathrm{c} 1}^{(0)}<h<h_{\mathrm{c} 2}^{(0)}$, and hence act as impenetrable barriers to QPs (see below for a detailed discussion of the physics of local moments). Therefore the problem of the response of the magnetic system to site dilution is analogous to that of the evolution of a Bose-Einstein condensate upon increasing the strength of a random potential in which the condensate is immersed. For a strong enough random potential the bosonic system will be fragmented into Anderson-localized states, sitting in the rare regions which are devoid of vacancies. This phase is called Bose glass in the literature of disordered interacting bosons [26]. Given that we are effectively working in the grand-canonical ensemble, the transition from a condensed phase to a Bose glass is accompanied by a decrease in the population, due to the fact that disorder lowers dramatically the local chemical potential in the regions close to 
impurities. This same localization-condensation transition can be probed by varying the field at fixed disorder strength: in this case the transition is clearly understood as a reduction of the density of the QPs/QHs, induced by the decrease/increase of the magnetic field. When traversing this transition in the reversed sense, by driving it with either a field or with disorder, we observe that the region occupied by the QPs/QHs undergoes a quantum percolation transition from a localized disordered phase, in which only disconnected rare clean regions host QPs/QHs, to a percolated ordered phase, in which the regions hosting QPs/QHs connect to form a percolating network. This geometric transition can be directly visualized by calculating the local magnetization profile, as shown in figure 6 . The novel, percolative nature of this transition with respect to the condensation in the clean case reveals itself in the critical exponents, which can be determined numerically via finite-size scaling $[11,12]$. The resulting values are quite different from those of the transition in the clean system, and in particular it is found that the dynamical critical exponent $z$ equals the spatial dimension $d$, which is consistent with an early theoretical prediction [26].

From the point of view of macroscopic observables, the Bose glass is fundamentally dominated by the absence of a gap in its excitation spectrum. Indeed in this phase the system is fragmented into droplets of QPs/QHs that are hosted on rare, but arbitrarily large regions, and which can therefore support arbitrarily low-energy excitations, in the form of localized magnon excitations analogous to those appearing in the ordered phase in the clean system. This means that the system displays a finite response to an applied magnetic field, namely its uniform susceptibility remains finite (corresponding to a finite compressibility of the QPs/QHs) even in the absence of long-range coherence. This is clearly illustrated in figure 7.

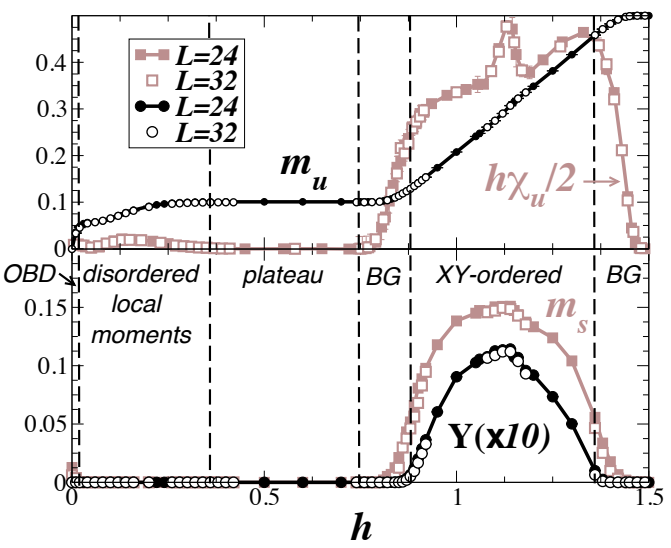

Figure 7. Zero-temperature field scan for the site-diluted Heisenberg bilayer with $J / J^{\prime}=8$ and $p=0.2$, from Roscilde et al., [11].

So far we have worked under the assumption that dangling spins left unpaired by site dilution are fully polarized by the field. Actually the evolution of their magnetic state represents another very fascinating aspect of the physics of these systems. Indeed, as already mentioned in the previous sections, in zero field dilution liberates local $S=1 / 2$ moments (LMs) which are exponentially localized close to the site of an unpaired spin and which interact with each other via the effective couplings of equation (4). These couplings, although weak, are sufficient for the LMs to order antiferromagnetically at experimentally relevant temperatures [27]. Hence, at any finite dilution concentration smaller than the classical percolation threshold, the zero-field ground state displays long-range antiferromagnetic order (order-by-disorder phase), (see figure 8).

Yet the long-range order appearing in this phase can be easily destroyed by a small field in a quite peculiar way $[11,12]$. Indeed the system of LMs features highly inhomogeneous couplings, and hence the response to the field is equally diverse. In the case of low dilution (to which this discussion is restricted) most of the LMs are weakly coupled to the other nearby LMs which sit at a distance of the order of the average inter-vacancy spacing $p^{-1 / d}$; but a minority of the LMs might be involved in a LM dimer (with probability $\sim p^{2}$ ), in a trimer (with probability $\sim p^{3}$ ), and so on. 

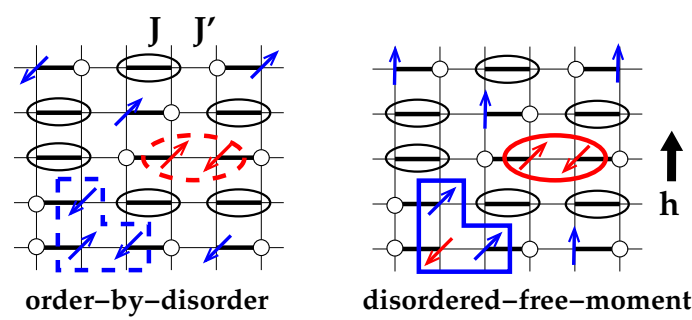

disordered-free-moment

Figure 8. Sketch of the quantum phase transition between the order-by-disorder phase and the disordered-local-moment phase in a diluted coupled-dimer system. Left panel: At zero applied field, spins on intact dimers form singlets (solid ellipses), dimers of local moments (LMs) have a strong singlet component (dashed ellipse), whereas the other LMs (blue arrows) participate in the order-by-disorder state. Right panel: Upon applying a field, the LMs are mostly polarized, but local singlets and localized down-spins can survive on clustered LMs, leading to the disordered LM phase.

This means that when the field is strong enough to overcome the typical coupling energy between two LMs, it polarizes a majority of LMs destroying thereby their long-range magnetic order. Yet, in this disordered $L M$ phase, a minority of strongly coupled LMs resist polarization, and they host locally one or more spin which is polarized opposite to the field. In particular, a mapping of the localized $S=1 / 2$ spins which remain antiparallel to the field onto hardcore bosons, reveals for the disordered LM phase a clear nature of a Bose glass, characterized by fragmentation of a Bose gas into disconnected localized states. This can be quantitatively confirmed by a finite-size scaling study of the transition between the order-by-disorder phase and the disordered LM phase: the extracted critical exponents turn out to be fully consistent with those of the 2D superfluid-to-Bose-glass transition [12]. In particular, as in a Bose glass, the disordered LM phase is not fully polarized, and it remains gapless, given that all different clusters of strongly coupled LMs see a different magnetic environment and hence exhibit a different local gap to further polarization. Nonetheless the local gaps are distributed around some distinct values associated with LM dimers, trimers, etc. These values manifest themselves in a special form of the magnetization curve, exhibiting pseudoplateaus which mark the accomplished polarization of one class of LM clusters, (see figure 9).

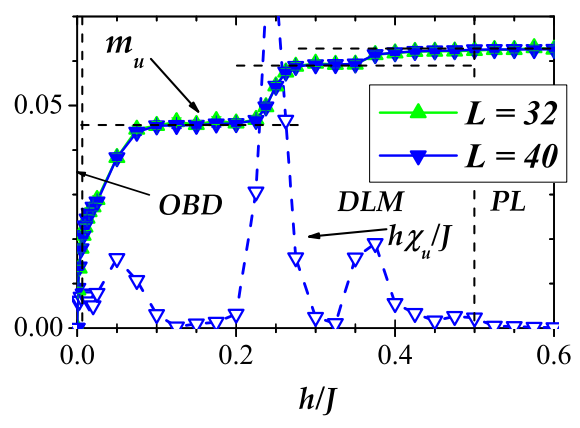

Figure 9. Magnetization and susceptibility curve of a planar array of dimers with $J / J^{\prime}=4$ and dilution $p=1 / 8$, showing a characteristic succession of pseudo-plateaus.

If the coupling ratio $J / J^{\prime}$ is large enough, or the disorder concentration is weak enough, upon further increasing the field, the LMs can be fully polarized at a field $h<h_{\mathrm{c} 1}^{(0)}$, namely before the magnetization process of the intact dimers begins. Under this assumption, a gapped plateau phase (characterized by a marked plateau of the uniform magnetization at the value $m=p / 2$ ) appears, completely separating the physics of the LMs from that of the intact dimers. On the other hand, 
for smaller $J / J^{\prime}$ ratios or strong dilution the plateau phase might be completely washed out, and dimers start being magnetized in the Bose glass phase when LMs have not yet been fully polarized. In this case disordered LM phase and Bose glass phase coexist: indeed the Bose glass phase will show both localized triplet QPs in rare regions devoid of impurities, and localized anti-parallel spins in the complementary regions exhibiting clustering of impurities.

\section{Conclusions}

In conclusion, large-scale quantum Monte Carlo simulations give access to the vast wealth of novel quantum phases appearing in quantum magnets when quantum fluctuations are tuned to a critical strength in the presence of disorder in the magnetic lattice. These novel phases are the result of the separation, induced by quantum fluctuations, between the geometric percolation of the lattice and the loss of long-range magnetic order. Their common denominator is a rich structure of the low-energy spectrum, with the absence of a gap and a possibly anomalous role of the low-energy excitations in the response functions.

This rich physics enjoys the availability of a large family of $S=1 / 2$ compounds exhibiting strongly fluctuating quantum magnetism, and which are amenable to dilution of the magnetic lattice by non-magnetic doping. In particular, candidate systems are spin-ladder materials such as $\mathrm{Cu}_{12}\left(\mathrm{C}_{5} \mathrm{H}_{12} \mathrm{~N}_{2}\right)_{2} \mathrm{Cl}_{4}$ [28] and $\left(\mathrm{C}_{5} \mathrm{H}_{12} \mathrm{~N}_{2}\right)_{2} \mathrm{Cu} \mathrm{Br}_{4}$ [29], planar dimer systems such as $\mathrm{Sr}_{2} \mathrm{Cu}\left(\mathrm{BO}_{3}\right)_{2}$ [30], magnetic bilayer compounds such as $\mathrm{BaCuSi}_{2} \mathrm{O}_{6}$ [31]. In all these compounds the $S=1 / 2$ spins is carried by a $\mathrm{Cu}^{2+}$ ion, which can be replaced by non-magnetic $\mathrm{Mg}^{2+}$ and $\mathrm{Zn}^{2+}$ to give site dilution of the lattice. Alternatively bond disorder can be realized by chemical substitution of the non-magnetic ions bridging the magnetic couplings $[32,33]$. As concerns specifically the physics of magnetic BEC in the presence of disorder, analogous phenomena to those observed in $S=1 / 2$ compounds can be also recovered with $S=1$ Haldane chains, or with $S=1$ antiferromagnets with strong single-ion anisotropy [34]. We therefore believe that quantum magnets under static doping offer the possibility of tremendously deepening our understanding of quantum many-body physics in a controlled random environment.

\section{Acknowledgements}

We thank Omid Nohadani for useful discussion. This work was supported by DOE through grant No. DE-FG02-06ER46319. The numerical computations were carried out on the University of Southern California high-performance computer cluster.

\section{References}

1. Sondhi S.L., Girvin S.M., Carini J.P., Shahar D., Rev. Mod. Phys., 1997, 69, 315.

2. Giamarchi T., Rüegg C., Tchernyshyov O., Nat. Phys., 2008, 4, 198.

3. Vajk O.P. et al., Science, 2002, 295, 1691.

4. Sandvik A.W., Phys. Rev. B, 2002, 66, 024418.

5. Vajk O.P., Greven M., Phys. Rev. Lett., 2002, 89, 177202.

6. Sandvik A.W., Phys. Rev. Lett., 2002, 89, 177201.

7. Sknepnek R., Vojta T., Vojta M., Phys. Rev. Lett., 2004, 93, 097201.

8. Yu R., Roscilde T., Haas S., Phys. Rev. Lett., 2005, 94, 197204.

9. Roscilde T., Haas S., Phys. Rev. Lett., 2005, 95, 207206.

10. Roscilde T., Haas S., J. Phys. B 2006, 39, 153.

11. Roscilde T., Phys. Rev. B, 2006, 74, 144418.

12. Yu R., Roscilde T., Haas S., New J. Phys., 2008, 10, 013034.

13. Lee P.A., Ramakrishnan T.V., Rev. Mod. Phys., 1985, 57, 287.

14. Sigrist M., Furusaki A., J. Phys. Soc. Jpn., 1996, 65, 2385.

15. Mikeska H.-J., Ghosh A., Kolezhuk A.K., Phys. Rev. Lett., 2004, 93, 217204. 
16. Griffiths R.B., Phys. Rev. Lett., 1969, 23, 17;

McCoy B.M., Phys. Rev. Lett., 1969, 23, 383;

McCoy B.M., Phys. Rev., 1969, 188, 1014.

17. Weichman P.B. Preprint arXiv:0810.3263, 2008.

18. Syljuåsen O.F., Sandvik A.W., Phys. Rev. E, 2002, 66, 046701.

19. Manousakis E., Rev. Mod. Phys., 1991, 63, 1.

20. Stauffer D., Aharony A. Introduction to Percolation Theory. Taylor and Francis, London, 1994.

21. Sandvik A.W., Scalapino D.J., Phys. Rev. Lett., 1994, 72, 2777.

22. Roscilde T., Haas S., Phys. Rev. Lett., 2007, 99, 047205.

23. Dagotto E., Rice T.M., Science, 1996, 271, 618.

24. Yu R., Roscilde T., Haas S., Phys. Rev. B, 2006, 73, 064406.

25. Matsumoto M. et al., Phys. Rev. B, 2001, 65, 014407.

26. Fisher M.P.A. et al., Phys. Rev. B, 1989, 40, 546.

27. Azuma M. et al., Phys. Rev. B, 1997, 55, R8658.

28. Chaboussant G. et al., Eur. J. Phys. B ,1998, 6, 167.

29. Watson B.C. et al., Phys. Rev. Lett., 2001, 86, 5168.

30. Sebastian S.E. et al., Phys. Rev. B, 2005, 71, 212405.

31. Sebastian S.E. et al., Phys. Rev. B, 2005, 72, 100404.

32. Oosawa A., Tanaka H., Phys. Rev. B, 2002, 65, 184437.

33. Masuda T., Zheludev A., Uchinokura K., Chung J.H., Park S., Phys. Rev. Lett., 2004, 93, 077206.

34. Paduan-Filho A. et al., Phys. Rev. B, 2004, 69, 020405; Zapf V. et al., Phys. Rev. Lett., 2006, 96, 077204.

\title{
Виявлення нових квантових фаз у квантових антиферомагнетиках на випадкових гратках
}

\author{
Р. Йу ${ }^{1,2}$, С. Гаас ${ }^{3}$, T. Рошільд ${ }^{4}$ \\ 1 Відділ фізики і астрономії, Університет Теннессі, Ноксвілл, TN 37996, США \\ 2 Відділення матеріалознавства і технології, Національна лабораторія в Оук Рідж, \\ Оук Рідж, TN 32821, США \\ 3 Відділ фізики і астрономії, Університет Південної Каліфорнії, Лос-Анджелес, СА 90089-0484, США \\ 4 Лабораторія фізики, Еколь нормаль Сюпер'єр в Ліоні, алея д'Італі 46, 69003 Ліон, Франція
}

Отримано 5 травня 2009 р.

\begin{abstract}
Квантові магнетики є ідеальним майданчиком для контрольованої реалізації нових квантових фаз і квантових фазових переходів. 3 гамільтоніаном системи можна справді маніпулювати, прикладаючи магнітне поле або тиск до зразка. Коли допувати систему немагнітними домішками, з'являються нові неоднорідні фази через конкуренцію між геометричною випадковістю і квантовими флуктуаціями. У цій статті ми робимо огляд нашої недавньої праці з квантових фазових переходів і нових квантових фаз, які реалізуються у невпорядкованих квантових магнетиках. Виявлено, що неоднорідність системи сильно впливає на фазові переходи, змінюючи їх клас універсальності, і приводить до нової квантово-перколяційної природи переходу. Такі переходи пов'язують звичайні впорядковані фази з незвичайними квантовими невпорядкованими фазами - квантовими фазами Гріфітса, фазами магнітного бозе-скла, які мають безщілинний спектр, зв'язаний з низькоенергетичними локалізованими збудженнями.
\end{abstract}

Ключові слова: антиферомагнетики Гайзенберга, квантовий безлад, геометрична випадковість, перколяція, бозе-скло

PACS: $75.10 . J m, 75.10 . N r, 75.40 . C x, 64.60 . A k$ 